\title{
WHO HAS Got THE SMARTS?
}

\author{
William C. McGrew
}

School of Psychology and Neuroscience, University of St. Andrews, Scotland.

wcm21@cam.ac.uk

A Review of the Book

Are We Smart Enough to Know How Smart Animals Are?

By Frans de Waal. 2016.

Granta, London, 340 pages.

ISBN 978-1-78378-304-5 (Hardback, £14.99)

Over almost 30 years, Frans de Waal has produced a string of stimulating and informative books on a variety of topics in animal behaviour, starting with his prizewinning Chimpanzee Politics (1982). All were founded on his training in the 'classical' ethology of the Dutch school, having been mentored by Garard Baerends and Jan van Hooff. All have featured as mainstays our nearest living relations of the genus Pan, that is, chimpanzee and bonobo, which inevitably calls for comparison with ourselves. For these two basic reasons, his latest book, like its predecessors, should be of interest to readers of Human Ethology Bulletin.

The new book's topic is intelligence, or as de Waal prefers to put it, evolutionary cognition. This follows nicely on the subjects of his earlier books, which have looked at politics, cooperation, empathy, culture, etc., in species other than ourselves. But de Waal is careful not to fall into the trap of dichotomous comparison, that is, human versus nonhuman. Rather, he covers a wider range of species to greater depth, than did his earlier, more primatocentric books. Chimpanzees still carry the load, but especially impressive are his treatments of elephant, octopus, and corvids.

As always, his books follow an admirable format: Readable, entertaining yet fact-filled prose, with superscripts rather in-text citations. The superscripts lead to a 13-page set of notes, which are mostly citations, and these in turn lead to a 28-page bibliography and a 14-page, detailed index. There is even a bonus, in the form of a 54-item glossary of scientific jargon terms. The result is that anything claimed by de Waal is readily available 
for background verification, unlike the work of so many other popularisers of science. As for the author's own illustrations, suffice it to say that perhaps his talents lie more with words than with pictures.

So, what about the content? The first three chapters are scene-setters, providing historical background for current research. Present-day human ethologists who come from outwith zoology will learn a lot about sub-discipline's roots, with appropriate homage paid to founding figures, such as Griffin, Lorenz, Tinbergen, Uexkuell, etc. As usual, de Waal is also cosmopolitan in outlook, with credit given to pioneers of Japanese primatology, especially Imanishi. He presents a useful reprise of the battles between American behaviourists and European ethologists for the soul of animal behaviour, which eventually led to a mutually-useful synthesis. Finally, he reminds us that comparative studies of cognition need to go beyond the primates, beyond the mammals, and even beyond the vertebrates. That is, true comparative psychology needs to be graduated along a continuum, rather than divided by a crude binary choice.

Chapters 4-8 are the meat of the book. Communication, and especially language, about which he is sceptical, in any species of but humans, is more complicated than the usual treatments by advocates. He uses ape and parrot studies aptly. A range of complex cognition is tackled, from mechanisms of social learning (e.g. imitation) to theory of mind (which he prefers to call perspective taking), to empathy, to culture. Prominent in all his arguments is the importance of sound methods, both valid and reliable. Experimental designs that compare apes and humans on a non-level playing field, such that humans gain a specious advantage, are dissected and revealed in their speciesist bias. On social skills, readers of de Waal are on familiar ground: reconciliation, consolation, tactical deception, triadic awareness, cooperation, inequity, etc. Mental time-travel gets a chapter, both backward (episodic memory) and forward (advance planning), while another chapter focuses on the self, from mirror-image recognition to delayed gratification.

So far, so good, but what about problems? One of de Waal's greatest strengths has always been his story-telling, that is, illustrative, sometimes mind-stretching examples, often as case studies, or even as one-off events. These come across as telling and memorable, but are they valid? That is, is an anecdote an instance of a more general phenomenon, or instead, could it be an accident, coincidence, mistake, misunderstanding, or even a hoax? That is, of these six possibilities, only one will turn out to be scientifically useful, and on top of that, anecdotes often tend to end up being misused (Sarringhaus et al. 2005). He is right to say that such single cases should be published, if for no other reason than to alert fellow researchers to the possibility of their existence, but often one-off cases raise more questions than they answer.

Another problem is coverage. None of us is able to be equally well-informed on a first-hand basis about research done in nature and captivity, by observation and experiment, over a wide range of organisms. De Waal is primarily a student of captive subjects, whose claim to fame was to let them inform us by their spontaneous behaviour, the validity of which is enhanced by housing and keeping them in environments that are as ecologically valid as possible. (His one foray into meeting chimpanzees in the wild, at Mahale in Tanzania, is amusingly and candidly reported here. For an extended version, 
see de Waal, 2003). But this means that he sometimes slips up on secondary reporting of the details of actitivities in nature, such as ape elementary technology, for example confusing tool kits and tool sets. However, these are minor quibbles, bordering on the pedantic.

A measure of a good thesis, well-argued, is the extent to which its conclusion points us to future advances. The author's 11-page summing-up is masterly, raising our expectations. He began with a basic, essential proposition that goes back to Darwin: Our abiding null-hypothesis should be evolutionary continuity, with the onus on scientists to show discontinuity, rather than starting from the stance of human special status. Results published in recent months, since the appearance of the book, attest to de Waal's foresight: Apes en masse passing the false-belief task, therefore showing theory of mind (Krupenye et al. 2016), wild capuchin monkeys spontaneously making (but not using) flaked stone tools (Proffitt et. al 2016), sculpting of hook tools extended to another species of crow (Rutz et al. 2016), wild chimpanzees collectively accumulating apparently functionless but striking cairns of stones (Kuehl et al. 2016), wild chimpanzees teaching offspring to fish for termites, via scaffolding demonstrations (Musgrave et al. 2016). The outpouring of new knowledge of evolutionary cognition predicted by de Waal is underway, and he has alerted us to it.

\section{ABOUT THE AUTHOR}

W.C. McGrew is Honorary Professor, School of Psychology and Neuroscience, University of St. Andrews. He has done ethology of human and non-human primates for more than 50 years. Articles published or in press for the last 12 months have been on chimpanzees and monitor lizards, Japan's contribution to cultural primatology, vertical bipedality in capuchin monkeys, evolution of non-human primate diet, and meeting and parting in wild chimpanzees.

\section{REFERENCES}

De Waal, F.B.M. (2003) Losing ten pounds to gain knowledge. Pan Africa News, 10, 17-19.

Krupenye, C., Kano, F. Hirato, S., Call, J., Tomasello, M. (2016) Great apes anticipate that other individuals will act according to false beliefs. Science, 354, 110-114. DOI

Kuehl, H.S. et al. (2016) Chimpanzee accumulative stone throwing. Scientific Reports, 6, 22219. $\underline{\mathrm{DOI}}$

Musgrave, S. et al. (2016) Tool transfers are a form of teaching among chimpanzees. Scientific Reports, 6, 34783. DOI

Proffitt, T. et al. (2016) Wild monkeys flake stone tools. Nature, 539, 85-88. DOI

Rutz, C. et al. (2016) Discover of species-wide tool use in the Hawaaian crow. Nature, 537, 403407. DOI

Sarringhaus, L.A. et al. (2005) Misuse of anecdotes in primatology: Lessons from citation analyses. American Journal of Primatology, 65, 283-288. 